\title{
Concerning the occurrence pattern of flux transfer events on the dayside magnetopause
}

\author{
D. G. Sibeck \\ Code 674, NASA/GSFC, 8800 Greenbelt Rd, Greenbelt, MD 20771, USA
}

Received: 16 October 2008 - Revised: 6 January 2009 - Accepted: 27 January 2009 - Published: 23 February 2009

\begin{abstract}
We present an analytical model for the magnetic field perturbations associated with flux transfer events (FTEs) on the dayside magnetopause as a function of the shear between the magnetosheath and magnetospheric magnetic fields and the ratio of their strengths. We assume that the events are produced by component reconnection along subsolar reconnection lines with tilts that depend upon the orientation of the interplanetary magnetic field (IMF), and show that the amplitudes of the perturbations generated during southward IMF greatly exceed those during northward IMF. As a result, even if the distributions of magnetic reconnection burst durations/event dimensions are identical during periods of northward and southward IMF orientation, events occurring for southward IMF orientations must predominate in surveys of dayside events. Two factors may restore the balance between events occurring for northward and southward IMF orientations on the flanks of the magnetosphere. Events generated on the dayside magnetopause during periods of southward IMF move poleward, while those generated during periods of northward IMF slip dawnward or duskward towards the flanks. Due to differing event and magnetospheric magnetic field orientations, events that produce weak signatures on the dayside magnetopause during intervals of northward IMF orientation may produce strong signatures on the flanks.
\end{abstract}

Keywords. Magnetospheric physics (Magnetopause, cusp, and boundary layers; Magnetosheath; Solar windmagnetosphere interactions)

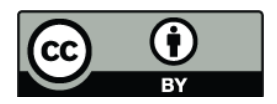

Correspondence to: D. G. Sibeck (david.g.sibeck@nasa.gov)

\section{Introduction}

Flux transfer events (FTEs) are common in the vicinity of the dayside magnetopause, where they can be identified on the basis of transient ( $\sim 1-2 \mathrm{~min})$ bipolar magnetic field signatures normal to the nominal magnetopause centered on magnetic field strength enhancements (Russell and Elphic, 1978). Because they tend to occur for southward IMF orientations (Berchem and Russell, 1984) and exhibit a mixture of magnetosheath and magnetospheric plasmas (Paschmann et al., 1982), FTEs are usually interpreted in terms of magnetic reconnection. Event motion and topology are consistent with generation along one or more parallel reconnection lines passing through the vicinity of the subsolar magnetopause with tilts dependent upon the IMF orientation (Rijnbeek et al., 1984; Daly et al., 1984), leading to an interpretation of the events in terms of bursty reconnection along component reconnection lines (Russell et al., 1985).

Surprisingly, FTEs observed on the flanks of the magnetosphere show no tendency to occur preferentially for southward IMF orientations (Kawano and Russell, 1997a). To reconcile this finding with an interpretation in terms of magnetic reconnection, Kawano and Russell (1997b) considered three possible explanations: (1) FTEs observed on the flanks originate locally via reconnection between antiparallel magnetosheath and magnetospheric magnetic fields, (2) FTEs observed on the flanks originate via component reconnection on the dayside magnetopause for southward IMF orientations, but via antiparallel reconnection on the high-latitude magnetopause during periods of northward IMF orientation, and (3) FTEs on the flanks originate along component reconnection lines passing through the subsolar magnetopause for all IMF orientations, but some process prevents events generated during periods of northward IMF orientation from being observed on the dayside.

Kawano and Russell (1997b) dispensed with the first possibility by demonstrating that events observed on the flanks

Published by Copernicus Publications on behalf of the European Geosciences Union. 
show no tendency to occur for the strongly antiparallel magnetosheath and magnetospheric magnetic fields that would favor local reconnection. Although they could not rule out a source at high latitudes during intervals of strongly northward IMF orientation, they did demonstrate that events observed on the equatorial flank magnetopause during intervals when the IMF points slightly northward must originate along a dayside reconnection line.

With a picture of FTEs as small flux ropes generated by patchy, localized, reconnection advocated by Russell and Elphic (1978) in mind, Kawano and Russell (1997b) opted for the third possibility. They proposed that "re-reconnection" (Kan, 1988; Nishida, 1989) at multiple sites slows or stops the motion of interconnected magnetosheath and magnetospheric magnetic field lines and therefore prevents the detection of FTEs generated by component reconnection on the dayside magnetopause. Because few FTEs occur for northward IMF orientations within the local time range from 10:00 to 14:00 LT, they suggested that re-reconnection predominates within two hours of local noon during periods of northward IMF orientation. In this model, the absence of re-reconnection permits FTEs to be observed for northward (and southward) IMF orientations at earlier and later local times.

While various theories (Alexeev et al., 1998; Moore et al., 2002) and simulations (Dorelli et al., 2007) predict reconnection on the dayside magnetopause during periods of northward interplanetary and/or magnetosheath magnetic field orientation, and the evidence for such reconnection is compelling (Phan et al., 1996; Anderson et al., 1997; Chandler et al., 1999; 2008; Fuselier et al., 2000; Chandler and Avanov, 2003; Trattner et al., 2004; Oieroset et al., 2008), it is less certain that re-reconnection prevents FTEs from being observed there during periods of northward IMF orientation. Simultaneous reconnection (re-reconnection) at multiple sites is precisely the mechanism invoked to explain true flux ropes with symmetric bipolar magnetic field signatures normal to the nominal magnetopause during periods of southward IMF orientation (e.g., Lee and Fu, 1985; Raeder, 2006). It seems unlikely that the same mechanism explains their absence during periods of northward IMF.

Instead, we propose that the orientation of FTEs relative to the draped magnetosheath and magnetospheric magnetic fields makes them difficult to observe on the dayside magnetopause during periods of northward IMF orientation. To show this, we invoke the component reconnection model to determine the initial orientation of FTEs formed along single- or multiple component reconnection lines passing through the subsolar magnetopause. We demonstrate that the events generate far greater perturbations in the draped magnetosheath magnetic field during periods of southward IMF than they do during periods of northward IMF. By alternately invoking flux and pressure balance across the magnetopause to relate the shapes of events in the magnetosheath and magnetosphere, we then demonstrate that the events also generate greater perturbations in the draped magnetospheric magnetic field during periods of southward IMF. Even if the distributions of burst durations/event dimensions are similar for northward and southward IMF orientations, events occurring during intervals of southward IMF orientations predominate in statistical studies of events on the dayside magnetopause. We discuss reasons why the same might not be true on the magnetotail flanks.

\section{Event perturbations on the dayside magnetopause}

Both Farrugia et al. (1988) and Sonnerup et al. (1992) have addressed the plasma and magnetic field perturbations generated in the magnetosheath and magnetosphere by a cylindrical FTE moving along the magnetopause. Farrugia et al. (1988) treated the case of an FTE with an arbitrary elliptical cross-section moving through an incompressible fluid, i.e. consistent with the motion of the event through the ambient media at a velocity small compared to the sound and Alfvén speeds. Sonnerup et al. (1992) developed a linear theory for flow around slender two-dimensional events moving through compressible fluids at sub-, trans-, and supersonic and Alfvénic velocities. As we wish to consider FTEs with arbitrary cross-section and the differences between FTE and magnetosheath flow velocities are small for the two limiting cases that we wish to consider (nearly stationary newlygenerated FTEs formed between parallel reconnection lines on the subsolar magnetopause and aged FTEs advecting antisunward with the magnetosheath flow), we adopt the model presented by Farrugia et al. (1988).

In this model, FTEs can be detected because their passage perturbs the ambient media. As illustrated in Fig. 1, the displacement of the ambient magnetic field lines by a passing flux rope results in (1) a characteristic bipolar magnetic field signature normal to the nominal magnetopause $(+,-$ or,-+ $\left.\mathrm{B}_{n}\right)$, (2) a decrease in the component of the magnetic field perpendicular to the axis of the event $\left(B_{\perp}\right)$ on the flanks of the event, (3) an increase in the same component over the axis of the event, and consequently (4) a rotation of the draped magnetic field towards an orientation parallel to the event axis on the flanks of the event but a rotation of the draped magnetic field towards a direction perpendicular to its axis over the event. The perturbations that an FTE generates in the surrounding media depend upon the orientation of the event axis, the components of the ambient magnetic fields perpendicular to this orientation, and the shape of the event. Here we determine each in turn for events on the dayside magnetopause, referencing the scenario illustrated in Fig. 1.

\subsection{Event orientation}

We assume that FTEs form via bursts of reconnection along extended component reconnection lines passing through the vicinity of the subsolar magnetopause (e.g., Raeder, 2006) 
and that their axes therefore initially lie parallel to those lines. Cowley (1976) has shown that the reconnection lines (and therefore event axes) may exhibit any orientation lying between those of the magnetosheath and magnetospheric magnetic fields. The literature is replete with suggestions concerning the precise orientation of the reconnection line(s) as a function of magnetospheric and magnetosheath magnetic fields: they may lie along the locus of points where magnetopause currents peak (Alexeev et al., 1998) or alternatively along the locus of points where magnetosheath and magnetospheric magnetic fields exhibit the greatest reconnecting components (Moore et al., 2002), along the locus of points that maximizes the Alfvén speed characterizing the reconnection outflow (Swisdak and Drake, 2007), or parallel to the magnetopause current vector (Sonnerup, 1974; Gonzales and Mozer, 1974). The various models share one prediction crucial to the present study, namely that the inclination of the reconnection line(s) with respect to the ecliptic increases as the shear between the arbitrary magnetosheath and fixed northward magnetospheric magnetic field orientations decreases.

We chose to employ the Sonnerup/Gonzalez/Mozer option to set the orientation of the component reconnection line. This option leads to a convenient analytical expression for the orientation of the reconnection lines on a planar magnetopause as a function of the magnetosheath and magnetospheric magnetic field strengths and directions. The lines lie parallel to the geomagnetic equator during periods when the magnetosheath magnetic field points strongly southward, tilt far out of the ecliptic during periods when the magnetosheath magnetic field points northward, and run from southern dawn (dusk) to northern dusk (dawn) during periods when the magnetosheath magnetic field has a duskward (dawnward) component. In contrast to the other component reconnection line model variants, the Sonnerup/Gonzalez/Mozer variant predicts that for strongly northward IMF reconnection ceases altogether unless the magnetosheath and magnetospheric magnetic fields have nearly the same magnitude.

Consider the case of a planar magnetopause, a magnetospheric magnetic field that points due northward with a magnitude $B_{1}$, and a magnetosheath magnetic field with a magnitude of $B_{2}$ that makes an angle of $\theta$ degrees with the magnetospheric magnetic field. As long as $\cos \theta$ does not exceed $B_{2} / B_{1}$, the component merging model predicts reconnection along a line passing through the subsolar point that makes an angle $\theta_{1}$ with the magnetospheric magnetic field, where $\theta_{1}$ is given by

$\theta_{1}=\sin ^{-1}\left(\left(B_{1} / B_{2}-\cos \theta\right) /\left(\left(B_{1} / B_{2}-\cos \theta\right)^{2}+\sin ^{2} \theta\right)^{1 / 2}\right)(1)$

When $\cos \theta>B_{2} / B_{1}$, the reconnection line does not lie between the two magnetic fields, and component reconnection becomes geometrically impossible. Equivalently, reconnection is impossible for weak northward magnetosheath magnetic fields in the Sonnerup (1974) variant. Reconnection can

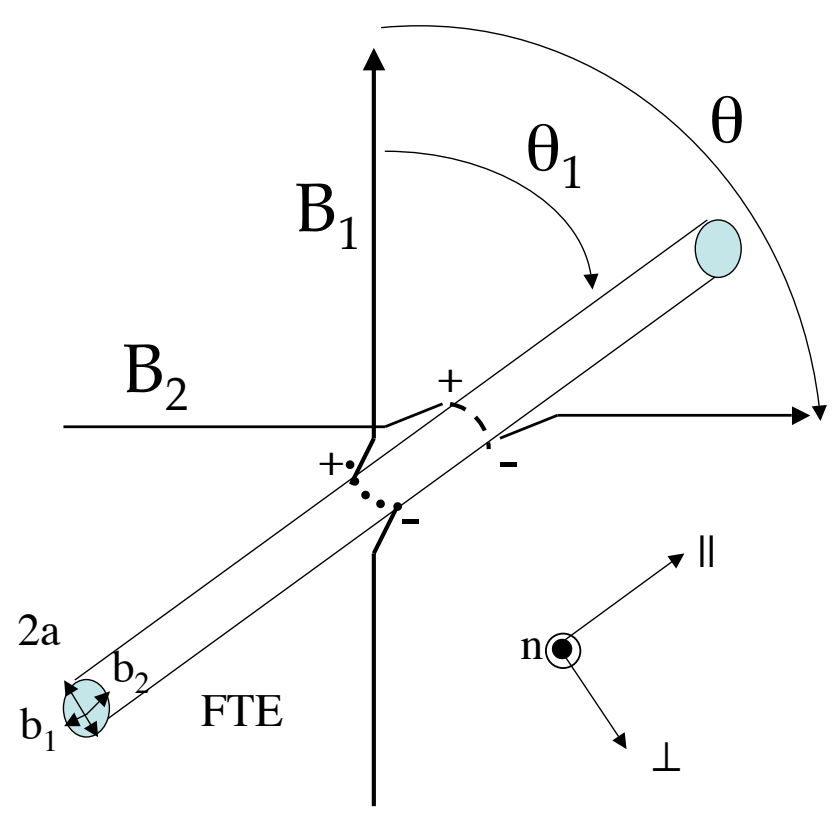

Fig. 1. A view of the dayside magnetopause from the Sun. The shear angle between the magnetosheath $\left(B_{2}\right)$ and magnetospheric $\left(B_{1}\right)$ magnetic fields is $\theta$, whereas the angle between the axis of the FTE and the magnetospheric magnetic field is $\theta_{1}$. The northward magnetospheric magnetic field drapes under, whereas the duskward magnetosheath magnetic field drapes over, a flux transfer event whose axis runs from southern dawn to northern dusk. The draped field gains a positive $\left(+B_{n}\right)$ component normal to the nominal magnetopause in both the magnetosheath and the magnetosphere on the northern edge of the event, but a negative $\left(-B_{n}\right)$ component on the southern edge. During the encounter with the event, the magnetosheath magnetic field veers southward (dashed curve), while the magnetospheric magnetic field backs dawnward (dotted curve). Draped magnetic field strengths exceed those in the ambient media. The FTE has a dimension of $2 a$ in the plane of the magnetopause, protrudes a distance $b_{1}$ into the magnetosphere, and a distance $b_{2}$ into the magnetosheath.

continue along highly tilted reconnection lines for the other component reconnection line model variants.

\subsection{The components of the ambient magnetic fields per- pendicular to the event axis}

The component of the magnetospheric magnetic field perpendicular to the event axis is given by $B_{1} \sin \theta_{1}$, while the component of the magnetosheath magnetic field perpendicular to the event axis is given by $B_{2} \sin \left(\theta-\theta_{1}\right)$.

\subsection{FTE dimensions and perturbations}

Consider the case of an elongated FTE with an elliptical cross-section whose major axis has a dimension of $2 a$ in the plane of the magnetopause, but whose semi-minor axis protrudes a distance $b_{1}$ into the magnetosphere. Solutions for the draped magnetic field strength and direction parallel those for 

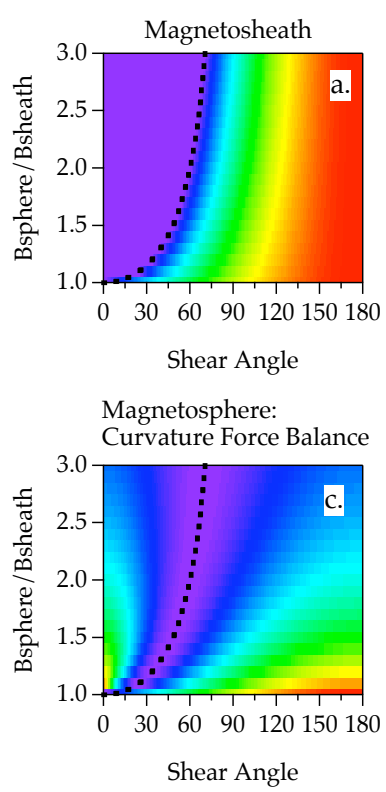

Fig. 2. Ratios of perturbations in the magnetic field component perpendicular to the event axis in the plane of the magnetopause to ambient magnetic field strengths as a function of the ratio of magnetospheric to magnetosheath magnetic field strengths and the shear angle between the magnetospheric and magnetosheath magnetic fields: (a) in the magnetosheath for an event with a circular magnetosheath cross-section $\left(b_{2}=a\right)$, (b) in the magnetosphere for an event with a magnetospheric cross-section determined by flux balance, and (c) in the magnetosphere for an event with a magnetospheric cross-section determined by force balance across the magnetopause. According to the component reconnection model of Sonnerup (1974) and Gonzales and Mozer (1974), no reconnection or FTEs can occur in the region of parameter space above the dashed lines in each panel.

flow around an elliptical cylinder (Batchelor, 1979). The enhancement in the magnetospheric magnetic field component transverse to the event axis just outside the crest of the FTE $\left(\delta B_{1 \perp}\right)$ is given by

$\delta B_{1 \perp}=\left(b_{1} / a\right) B_{1} \sin \theta_{1}$,

while the component of the magnetic field normal to the nominal magnetopause $\left(\delta B_{1 n}\right)$ attains a peak value of

$\delta B_{1 n}=\left(0.5+b_{1} / 2 a\right) B_{1} \sin \theta_{1}$

at a distance $a^{2} /\left(a^{2}+b_{1}^{2}\right)^{1 / 2}$ along the magnetopause from the center of the ellipse and a distance $b_{1}^{2} /\left(a^{2}+b_{1}^{2}\right)^{1 / 2}$ normal to the magnetopause into the magnetosphere. Event amplitudes increase as the ratio $b_{1} / a$ increases, while the range of locations over which they can be observed increases with $b_{1}$.

Substituting $\theta_{1}$ from Eq. (1) into Eq. (3), we obtain an expression for the peak magnetic field strength normal to the nominal magnetopause in the magnetosphere as a function of the shape of the elliptical FTE, the magnetic field strengths
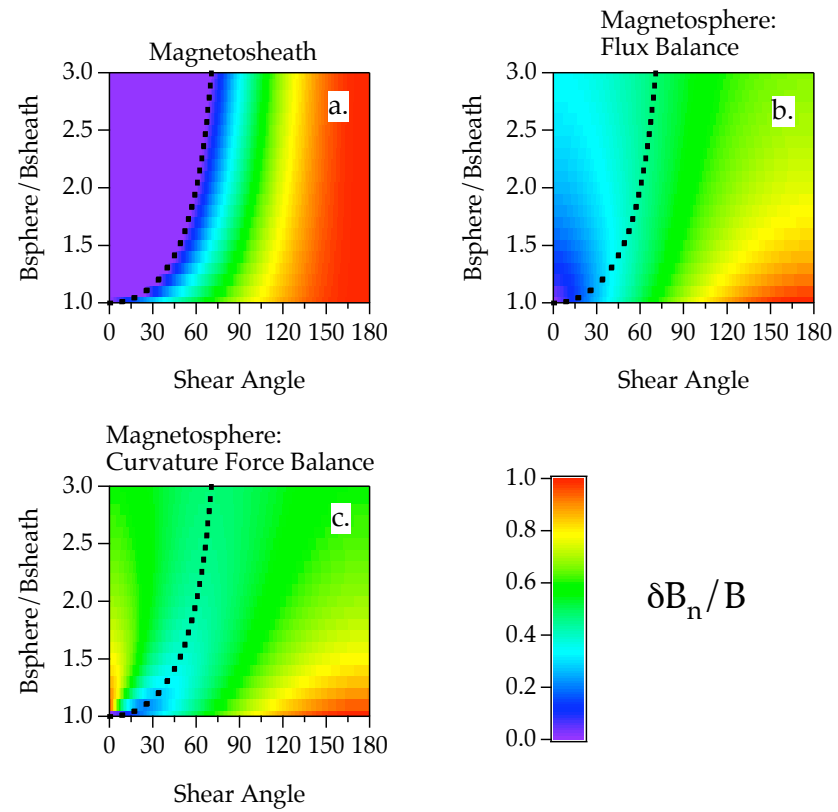

Fig. 3. As in Fig. 2, but for ratios of the magnetic field perturbations normal to the nominal magnetopause to the ambient magnetic field strengths.

on both sides of the boundary, and the angle between the northward magnetospheric magnetic field and the magnetosheath magnetic field:

$$
\begin{aligned}
\delta B_{1 n}= & \left(0.5+b_{1} / 2 a\right) B_{1}\left(B_{1} / B_{2}-\cos \theta\right) / \\
& \left(\left(B_{1} / B_{2}-\cos \theta\right)^{2}+\sin ^{2} \theta\right)^{1 / 2}
\end{aligned}
$$

A similar derivation for the peak magnetic field strength normal to the nominal magnetopause in the magnetosheath $\left.\delta B_{2 n}\right)$ gives:

$$
\begin{aligned}
\delta B_{2 n}= & \left(0.5+b_{2} / 2 a\right) B_{2}\left(\left(B_{1} \cos \theta / B_{2}-1\right)\right. \\
& \left(\left(B_{1} / B_{2}-\cos \theta\right)^{2}+\sin ^{2} \theta\right)^{1 / 2}
\end{aligned}
$$

where we have assumed that the dimension of the FTE along the magnetopause is the same (2a) in the magnetosheath and magnetosphere, but allowed for the possibility that the semiminor axis of the FTE protrudes a different distance, $b_{2}$, into the magnetosheath. Equations (1) and (2) can be used to derive corresponding expressions for the enhancements in the magnetic field components perpendicular to the event axis just outside the crests of the FTE in the magnetosphere and magnetosheath.

\subsection{Results for the magnetosheath}

Figures $2 \mathrm{a}$ and $3 \mathrm{a}$ present the ratios of predicted perturbation magnetosheath magnetic field components transverse to the event axis and normal to the nominal magnetopause to background magnetic field strengths for FTEs with circular $\left(b_{2}=a\right)$ cross-sections in the magnetosheath. As required by 
the magnetosheath versions of Eqs. (2) and (3), the two panels are identical. For any ratio of magnetospheric to magnetosheath magnetic field strengths, perturbations in the magnetosheath peak at $\delta B_{2 n}=\delta B_{2 \perp}=B_{2}$ when the magnetosheath magnetic field points due southward, i.e. the shear angle between the magnetosheath and magnetospheric magnetic fields is $180^{\circ}$. Perturbation amplitudes diminish with decreasing shear angle, somewhat slower for comparable magnetosheath and magnetospheric magnetic field strengths than for magnetospheric magnetic field strengths far greater than those in the magnetosheath. For equal magnetosheath and magnetospheric magnetic field strengths, $B_{n}$ amplitudes at shear angles of $90^{\circ}$ (i.e. ecliptic IMF orientations) are $70 \%$ of those at shear angles of $180^{\circ}$ (southward IMF orientations). As the ratio of magnetospheric to magnetosheath magnetic field strengths increases, the ratio of the perturbation to background magnetic field strength amplitudes diminishes. Note that in these and the other panels of Figs. 2 and 3, points above the dashed line lie within the region where component reconnection is forbidden.

\subsection{Results for the magnetosphere}

Although we maintain our assumption of circular event cross-sections in the magnetosheath $\left(b_{2}=a\right)$, we do not assume circular cross-sections in the magnetosphere $\left(b_{1} \neq a\right)$. To calculate the amplitudes of the signatures expected in the magnetosphere, we must therefore determine the distance that events protrude into the magnetosphere $\left(b_{1}\right)$ as a function of their semimajor axis $(a)$. We consider two approaches: one appropriate to times early in the development of the FTE when flux balance prevails, and the second to later times when pressure balance prevails.

The first approach acknowledges that FTEs form via the near-instantaneous reconnection of equal quantities of magnetosheath and magnetospheric flux. An event with a length $l$ along the reconnection line intercepts a magnetospheric flux $0.5 \pi l \sin \theta_{1} b_{1} B_{1}$ and a magnetosheath flux $0.5 \pi l \sin (\theta-$ $\left.\theta_{1}\right) b_{2} B_{2}$, yielding $b_{1}=b_{2} B_{2} \sin \left(\theta-\theta_{1}\right) /\left(B_{1} \sin \theta_{1}\right)$. Since the magnetic field strength in the magnetosphere exceeds that in the magnetosheath, the events do not extend as far into the magnetosphere as they do into the magnetosheath $\left(b_{1}<b_{2}\right)$. Figures $2 b$ and $3 b$ show the peak amplitudes of the perturbations in the magnetosphere for $b_{2}=a$, as assumed above. These panels demonstrate that the ratios of FTE perturbation to background magnetic field strengths in the magnetosphere are greatest for antiparallel magnetosheath and magnetospheric magnetic fields with similar strengths, but fall off rapidly as the magnetosheath magnetic field rotates northward and/or the ratio of magnetospheric to magnetosheath magnetic field strengths increases beyond $\sim 1.5$. As noted by Ding et al. (1991), with the exception of the special case when magnetosheath and magnetospheric magnetic field strengths are identical, the ratios of perturbation amplitudes to background magnetic field strengths are generally less in the magnetosphere (panels $2 b$ and $3 b$ ) than they are in the magnetosheath (panels $2 \mathrm{a}$ and $3 \mathrm{a}$ ).

In the second approach, we incorporate the effects of magnetic curvature forces. Long after formation, the FTE moves along the magnetopause with the magnetosheath velocity at an elevation relative to that boundary determined by the balance of forces across the magnetopause. In steady-state, the magnetohydrodynamic momentum equation can be expressed as:

$0=-\nabla\left(p+B^{2} / 2 \mu_{0}\right)+(\boldsymbol{B} \cdot \nabla) \boldsymbol{B}$

considering the component of this equation normal to the nominal planar magnetopause, setting the scale lengths of the gradients along the magnetopause in the direction of the background magnetic fields to be $2 a / \sin \theta_{1}$ or $2 a / \sin \left(\theta-\theta_{1}\right)$, but the scale lengths of the gradients along the direction normal to the magnetopause to be either $b_{1}$ or $b_{2}$ (as appropriate to the region under consideration), we obtain:

$$
\begin{aligned}
\left(p+B^{2} / 2 \mu_{0}\right)_{\text {in the event }}= & \left(p_{1}+B_{1}^{2} / 2 \mu_{0}\right)_{\text {far from the event }} \\
& +b_{1} B_{1} B_{1 n} \sin \theta_{1} / \mu_{0} a \\
= & \left(p_{2}+B_{2}^{2} / 2 \mu_{0}\right)_{\text {far from the event }} \\
& +b_{2} B_{2} B_{2 n} \sin \left(\theta-\theta_{1}\right) / \mu_{0} a
\end{aligned}
$$

where subscripts 1 and 2 refer to values in the corresponding regions (e.g., Paschmann et al., 1982). If the sum of the thermal and magnetic pressures far from the magnetopause are equivalent on both sides of the magnetopause, then:

$b_{1} B_{1} B_{1 n} \sin \theta_{1} / \mu_{0} a \approx b_{2} B_{2} B_{2 n} \sin \left(\theta-\theta_{1}\right) / \mu_{0} a$

where $B_{1}$ and $B_{2}$ are the unperturbed magnetospheric and magnetosheath magnetic field strengths and we will take $B_{1 n}$ and $B_{2 n}$ as the peak values for the perturbation magnetic fields just outside the events in both regions. By substituting values for $\theta_{1}$ from Eq. (1), $B_{1 n}$ and $B_{2 n}$ from Eqs. (4) and (5), and maintaining $b_{2}=a$ as noted above, one can obtain a quadratic expression for $b_{1}$ as a function of $a, \theta$, and the ratio of magnetospheric to magnetosheath magnetic field strengths. This expression yields a root:

$b_{1}=0.5 a\left(\left[1+8\left(\cos \theta-B_{2} / B_{1}\right)^{2} /\left(B_{1} / B_{2}-\cos \theta\right)^{2}\right]^{1 / 2}-1\right)(9)$

Substituting this value back into Eq. (2) and (3), we can determine the amplitude of signatures in the magnetosphere at times when the magnetic curvature forces applied to the FTE balance across the magnetopause. As can be seen in Figs. 2c and $3 \mathrm{c}$, force balance diminishes the amplitudes of perturbations in the magnetosphere from the initial values predicted by flux balance. This is because the strong magnetic curvature forces in the magnetosphere push events outward into the magnetosheath, weakening their magnetospheric signatures. Once again, the strongest magnetospheric signatures occur when there is a large shear between comparable magnetosheath and magnetospheric magnetic field strengths, i.e. during periods of southward IMF orientation. 


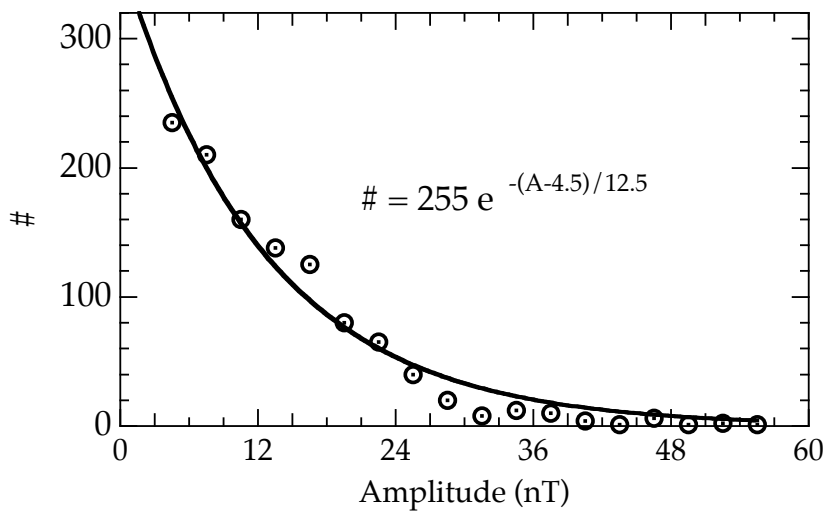

Fig. 4. Dots indicate the distribution of FTEs versus amplitude (in nT) observed by Cluster on the high-latitude and flank magnetopause (Wang et al., 2005). The solid curve shows a fit to the distribution.

\subsection{Occurrence patterns at the dayside magnetopause}

The results presented above quantify how the signatures of FTEs on the dayside magnetopause vary as a function of the ratio of magnetospheric to magnetosheath magnetic field strengths and the shear angle between the two magnetic field orientations. They indicate that FTEs generate greater perturbations in both the magnetosheath and the magnetosphere during periods when there is a high shear between the magnetosheath and magnetospheric magnetic fields than during periods when there is a low shear. Consequently, even if FTEs are equally common and attain similar dimensions for high and low shears, events occurring for southward IMF orientations will predominate in surveys of the dayside magnetopause. To determine the magnitude of this effect and for comparison with prior observations studies, we must identify the fraction of FTEs with amplitudes exceeding a specified detection threshold as a function of the shear angle $\theta$ between the magnetosheath and magnetospheric magnetic fields.

Figure 4 presents the distribution of FTE magnetic field strength perturbations (peak - surroundings) observed by Cluster on the flank and high-latitude dayside magnetopause as reported by Wang et al. (2005) and a fit given by

$N=255 e^{-(A-4.5) / 12.5}$

where $A$ is event amplitude and $N$ the number of events in each 3 nT bin. Wang et al. (2005) reported similar distributions for two other measures of event dimension: the peak-topeak amplitude of the bipolar magnetic field component normal to the nominal magnetopause and the time between these two peaks. Since the distribution of event dimensions must resemble those for event amplitudes and durations, there are many small but only a few large events.

For the purposes of this discussion, we assume that the distributions for the number of events versus event dimension are identical for all shears between the magnetosheath and magnetospheric magnetic field. This is equivalent to assuming that reconnection continues on the dayside magnetopause during periods of northward IMF orientation, and that the time-dependence of reconnection for northward IMF orientations is similar to that for southward IMF orientations. Then the distributions for the number of events versus event amplitude are similar for all shears, with the caveat that the amplitudes of the perturbations must be scaled to values appropriate for each shear. For example, an event with dimensions sufficient to generate a $10 \mathrm{nT}$ signature in the surrounding media for a $180^{\circ}$ shear between the magnetosheath and magnetospheric magnetic fields generates a much weaker perturbation for a low shear. According to Eq. (5), event amplitudes scale as $\sin \theta / 2$ for the singular case of identical magnetosheath and magnetospheric magnetic field strengths. If so, then the minimum dimension for event identification increases as $(\sin \theta / 2)^{-1}$ as $\theta$ diminishes below $180^{\circ}$. For the more general case in which magnetospheric magnetic field strengths exceed those in the magnetosheath, event amplitudes diminish even more rapidly as $\theta$ decreases (see Figs. 2a and $3 \mathrm{a}$ ) and therefore the minimum dimension for event identification also increases more rapidly.

Let the distribution of events versus amplitude for $180^{\circ}$ shear angles be that shown in Fig. 4. Then the number of events satisfying a $10 \mathrm{nT}$ selection threshold criterion at any shear angle $\theta$ is simply the area under the curve in Fig. 4 at amplitudes greater than $10 /(\sin \theta / 2) \mathrm{nT}$ for the singular case of identical magnetosheath and magnetospheric magnetic field strengths. The results shown in Fig. 5 indicate that $\sim 0 \%$ of the events exceed the threshold criterion during periods of due northward IMF $\left(\theta=0^{\circ}\right), \sim 30 \%$ during periods of ecliptic IMF $\left(\theta=90^{\circ}\right)$, and $\sim 45 \%$ during periods of strongly southward IMF $\left(\theta=180^{\circ}\right)$. For the more general case of unequal magnetosheath and magnetospheric magnetic field strengths, the percentage of events exceeding the threshold criterion at shears less than $180^{\circ}$ would be even lower. Thus, even if FTEs are equally common on the dayside magnetopause during periods of northward and southward IMF orientation, events occurring for southward IMF orientations will predominate in statistical surveys based on event signatures exceeding specified thresholds.

Kuo et al. (1995) reported the fraction of ISEE-1/2 passes through the dayside magnetopause exhibiting FTEs with peak-to-peak bipolar magnetic field amplitudes exceeding 10 $\mathrm{nT}$ as a function of IMF clock angle. As seen in Fig. 6, the percentage of events exceeding the threshold criterion is $\sim 0 \%$ during periods of strongly northward IMF, 30/60\% during periods of ecliptic IMF, and $\sim 55 \%$ during periods of strongly southward IMF orientation. Under an assumption that the dayside equatorial magnetospheric magnetic field pointed due northward during each of these events (i.e. that the IMF clock angle is the magnetopause shear angle), Fig. 6 can be compared directly with Fig. 5. Given the statistical uncertainties in the observational study, the two distributions are roughly comparable, indicating that few FTEs 


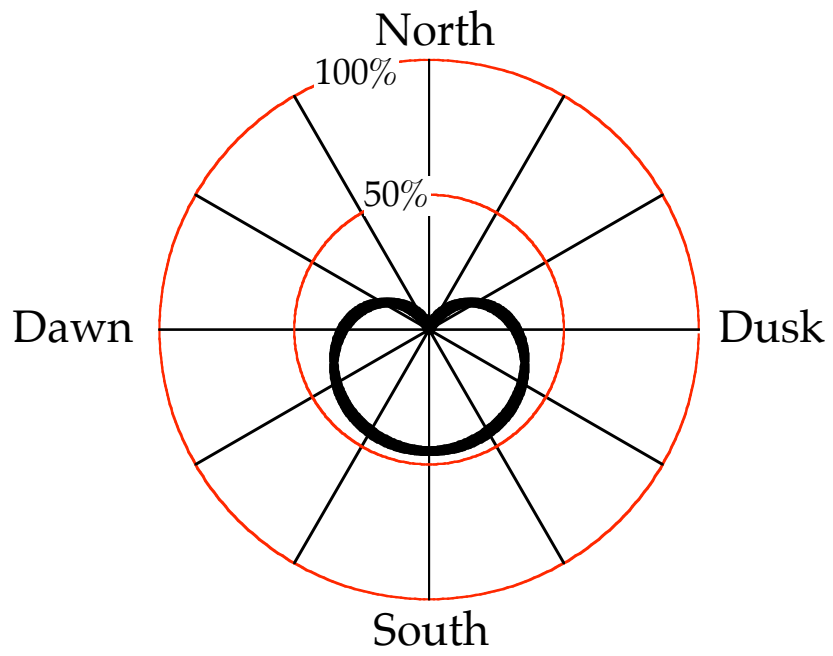

Fig. 5. The percentage of FTEs with perturbation amplitudes predicted to exceed $10 \mathrm{nT}$ as a function of the direction of the magnetosheath magnetic field orientation.

are identified on the dayside magnetopause during northward IMF/low shear conditions, but that the success rate increases to about $50 \%$ for southward IMF orientations. Since the theoretical distribution was calculated for an assumption that FTEs are equally common for high and low shears, the similarity of the two distributions indicates that FTEs may be present but go unnoticed on the dayside magnetopause during intervals of strongly northward IMF orientation.

\section{Summary and conclusion}

A desire to reconcile observations indicating that FTEs on the dayside magnetopause tend to occur for southward IMF orientations while those on the flanks do not motivated this paper. Rather than invoking re-reconnection in the vicinity of local noon to explain the absence of events on the dayside magnetopause during intervals of strongly northward IMF orientations, we sought an explanation in terms of magnetic field draping over the events. Under the specified assumptions that FTEs form along subsolar reconnection lines whose tilt depends upon the IMF orientation and then move slowly with respect to the surrounding magnetosheath and magnetospheric media, we derived simple analytical expressions for the maximum perturbations to be expected in the magnetic field component normal to the nominal magnetopause and the magnetic field component perpendicular to the event axis in the plane of the magnetopause.

Although we specified a circular cross-section for the events in the magnetosheath, we used flux balance to determine the corresponding elliptical cross-sections of the events in the magnetosphere. Consistent with previous simulation results, we found that events protrude much further into the magnetosheath than into the magnetosphere and gener-

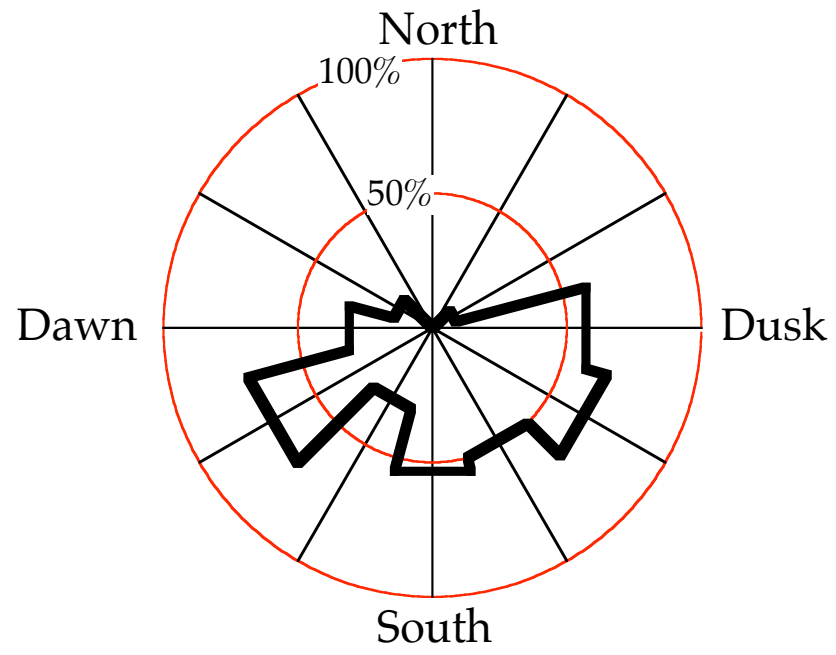

Fig. 6. The probability of FTE occurrence on an orbit regardless of region versus IMF orientation in the Y-Z GSM plane as reported by Kuo et al. (1995).

ate much larger perturbations relative to the magnetosheath magnetic field than to the magnetospheric magnetic field for the typical scenario in which magnetospheric magnetic field strengths exceed those in the magnetosheath. The discrepancy between magnetosheath and magnetospheric signatures becomes even greater under an assumption that pressure gradient and magnetic curvature forces balance across the magnetopause.

Consequently, there should be many more magnetosheath than magnetospheric FTEs. Consistent with this hypothesis, Kuo et al. (1995) reported 60\% more magnetosheath than magnetospheric FTEs on ISEE-1/2 dayside and flank magnetopause passes, Neudegg et al. (2000) and Wang et al. (2005) reported three times as many magnetosheath than magnetospheric FTEs on Equator-S dawn flank and Cluster high-latitude/flank passes, while Rijnbeek et al. (1984) reported only $\sim 33 \%$ more ISEE-1/2 dayside magnetosheath passes with events than magnetosphere passes. Nevertheless, Kawano and Russell (1996) reported almost equal numbers of the ISEE-1/2 events in the magnetosheath and magnetosphere. The discrepancy may result from differing event identification criteria or regions studied. For example, Kawano and Russell (1996) categorized a full one third of their events as occurring in an unclear domain, one in which it was not possible to determine whether the events occurred in the magnetosheath or the magnetosphere. This was a particular problem on the magnetotail flanks.

We presented our results for the amplitudes of FTE signatures as a function of the ratio of magnetospheric to magnetosheath magnetic field strengths and the shear angle between magnetosheath and magnetospheric magnetic field orientations. For fixed magnetosheath event cross-sections, events become more prominent in the magnetosphere as the 
magnetospheric magnetic field strength diminishes to magnetosheath levels. Event amplitudes in both the magnetosheath and magnetosphere peak for strongly antiparallel magnetosheath and magnetospheric magnetic field orientations. Since the dayside equatorial magnetospheric magnetic field points northward, events become more prominent during periods of southward interplanetary and magnetosheath magnetic field orientation. Statistical studies of events on the dayside magnetopause that employ minimum amplitude thresholds for event identification must therefore conclude that FTEs are more common for southward IMF orientations, even if transient bursts of reconnection are equally common for northward and southward IMF orientations.

The results presented in this paper provide an alternative, albeit partial, explanation for the prevalence of events on the dayside for southward IMF orientations, but similar event occurrence rates on the flanks for northward and southward IMF orientations. We suggest that events occurring for southward IMF orientations exhibit much stronger signatures than those occurring for northward IMF orientations on the dayside magnetopause in the immediate vicinity of local noon. The explanation is partial, because the question then arises as to why the same FTEs should be just as easily observable for northward and southward IMF orientations on the magnetotail flanks. Pending forthcoming work on the motion and orientation of FTEs, we speculate that events passing unnoticed on the dayside magnetopause may become more readily observable as they move towards the magnetotail flanks, because event, magnetospheric, and magnetosheath magnetic field orientations on the flanks all differ from those on the dayside magnetopause. Furthermore, magnetic curvature forces preferentially remove events occurring for southward IMF orientations via poleward motion over the mantles, leaving events occurring for northward IMF orientations to slip azimuthally over the flanks. These two factors result in enhanced rates of event occurrence for northward IMF orientations on the flanks with respect to the dayside magnetopause.

Finally, we should address our assumption that the events move slowly through ambient media that are incompressible. This is a reasonable assumption on the subsolar magnetopause for the first few moments after event formation between parallel subsolar reconnection lines, on the flank and high latitude magnetopause long after event generation when ambient flows sweep events downstream and magnetic curvature forces are weak, and on the magnetospheric side of the magnetopause where Alfvén and sound speeds are high. The assumption may be violated at intermediate latitudes or local times on the magnetosheath side if magnetic curvature forces succeed in accelerating events to speeds relative to the ambient magnetosheath flow comparable to or greater than the magnetosheath Alfvén and sound speeds.

The results presented by Sonnerup et al. (1992) provide a clue to the signatures expected for such trans- or super-sonic and Alfvénic event velocities. The components of the mag- netic field transverse and parallel to the event axis both increase. For supersonic and super-Alfvénic flows, the increase in the parallel component exceeds that for the transverse component, resulting in a magnetic field rotation towards the event axis. The same increase in the component parallel to the event axis affords an opportunity to detect event signatures even when shears between the magnetosheath and magnetospheric magnetic fields are small. However, since magnetosheath (and magnetospheric) events are identified primarily on the basis of bipolar magnetic field signatures normal to the nominal magnetopause, events moving at transor super-sonic velocities relative to the magnetosheath flow during periods of small shear would generally not be identified as FTEs. Consequently, the presence of such events does not affect our hypothesis that events occurring for southward magnetosheath or interplanetary magnetic field orientations must predominate in studies of FTEs on the dayside magnetopause because the signatures associated with FTEs occurring for northward IMF orientations are weak.

Acknowledgements. Topical Editor I. A. Daglis thanks C. Farrugia and another anonymous referee for their help in evaluating this paper.

\section{References}

Alexeev, I. I., Sibeck, D. G., and Bobrovnikov, S. Y.: Concerning the location of merging as a function of the magnetopause current strength, J. Geophys. Res., 103, 6675-6684, 1998.

Anderson, B. J., Phan, T.-D., and Fuselier, S. J.: Relationships between plasma depletion and subsolar reconnection, J. Geophys. Res., 102, 9531-9542, 1997.

Batchelor, G. K.: An Introduction to Fluid Dynamics, Cambridge Univ. Press, Cambridge, pp. 424-435, 1979.

Berchem, J. and Russell, C. T.: Flux transfer events on the magnetopause: Spatial distribution and controlling factors, J. Geophys. Res., 89, 6689-6703, 1984.

Chandler, M. O. and Avanov, L. A.: Observations at low latitudes of magnetic merging signatures within a flux transfer event during a northward interplanetary magnetic field, J. Geophys. Res., 108, 1358, doi:10.1029/2003JA009852, 2003.

Chandler, M. O., Fuselier, S. A., Lockwood, M., and Moore, T. E.: Evidence of component merging equatorward of the cusp, J. Geophys. Res., 104, 22623-22633, 1999.

Chandler, M. O., Avanov, L. A., Craven, P. D., Mozer, F. S., and Moore, T. E.: Observations of the ion signatures of double merging and the formation of newly closed field lines, Geophys. Res. Lett., 35, L10107, doi:10.1029/2008GL033910, 2008.

Cowley, S. W. H.: Comments on the merging of nonantiparallel magnetic fields, J. Geophys. Res., 81, 3455-3458, 1976.

Daly, P. W., Saunders, M. A., Rijnbeek, R. P., Sckopke, N., and Russell, C. T.: The distribution of reconnection geometry in flux transfer events using energetic ion, plasma and magnetic data, J. Geophys. Res., 89, 3843-3854, 1984.

Ding, D.-Q., Lee, L.-C., and Ma, Z.-W.: Different FTE signatures initiated by the bursty single $\mathrm{X}$ line reconnection and the multiple $\mathrm{X}$ line reconnection at the dayside magnetopause, J. Geophys. Res., 96, 57-66, 1991. 
Dorelli, J. C., Bhattacharjee, A., and Raeder, J.: Separator reconnection at Earth's dayside magnetopause under generic northward interplanetary magnetic field conditions, J. Geophys. Res., 112, A02202, doi:10.1029/2006JA011877, 2007.

Farrugia, C. J., Elphic, R. C., Southwood, D. J., and Cowley, S. W. H.: Field and flow perturbations outside the reconnected field line region in flux transfer events: Theory, Planet. Space Sci., 35, 227-240, 1988 .

Fuselier, S., Petrinec, S. M., and Trattner, K.: Cusp observations of high- and low-latitude reconnection for northward interplanetary magnetic field, J. Geophys. Res., 105, 253-266, 2000.

Gonzales, W. D., and Mozer, F. S.: A quantitative model for the potential resulting from reconnection with an arbitrary interplanetary magnetic field, J. Geophys. Res., 79, 4186-4194, 1974.

Kan, J. R.: A theory of patchy and intermittent reconnections for magnetospheric flux transfer events, J. Geophys. Res., 93, 56135623, 1988.

Kawano, H. and Russell, C. T.: Survey of flux transfer events observed with the ISEE 1 spacecraft: Rotational polarity and the source region, J. Geophys. Res., 101, 27299-27308, 1996.

Kawano, H. and Russell, C. T.: Survey of flux transfer events observed with the ISEE 1 spacecraft: Dependence on the interplanetary magnetic field, J. Geophys. Res., 102, 10307-11313, 1997a.

Kawano, H. and Russell, C. T.: Cause of postterminator flux transfer events, J. Geophys. Res., 102, 27029-27038, 1997 b.

Kuo, H., Russell, C. T., and Le, G.: Statistical studies of flux transfer events, J. Geophys. Res., 100, 3513-3519, 1995.

Lee, L.-C. and Fu, Z.-F.: A theory of magnetic flux transfer at the Earth's magnetopause, Geophys. Res. Lett., 12, 105-108, 1985.

Moore, T. E., Fok, M.-C., and Chandler, M. O.: The dayside reconnection X line, J. Geophys. Res., 107, 1332, doi:10.1029/2002JA009381, 2002.

Neudegg, D. A., Cowley, S. W. H., Milan, S. E., Yeoman, T. K., Lester, M., Provan, G., Haerendel, G., Baumjohann, W., Nikutowski, B., Büchner, J., Auster, U., Fornacon, K.-H., and Georgescu, E.: A survey of magnetopause FTEs and associated flow bursts in the polar ionosphere, Ann. Geophys., 18, 416-435, 2000, http://www.ann-geophys.net/18/416/2000/.
Nishida, A.: Can random reconnection on the magnetopause produce the low latitude boundary layer?, Geophys. Res. Lett., 16, 227-230, 1989.

Oieroset, M., Phan, T.-D., Angelopoulos, V., et al.: THEMIS multi-spacecraft observations of magnetosheath plasma penetration deep into the dayside low-latitude magnetosphere for northward and strong By IMF, Geophys. Res. Lett., 35, L17S11, doi:10.1029/2008GL033661, 2008.

Paschmann, G., Haerendel, G., Papamastorakis, I., Sckopke, N., Bame, S. J., Gosling, J. T., and Russell, C. T.: Plasma and magnetic field characteristics of magnetic flux transfer events, J. Geophys. Res., 87, 2159-2168, 1982.

Phan, T.-D., Paschmann, G., and Sonnerup, B. U. Ö.: Low-latitude dayside magnetopause and boundary layer for high magnetic shear 2. Occurrence of magnetic reconnection, J. Geophys. Res., 101, 7817-7828, 1996.

Raeder, J.: Flux Transfer Events: 1. generation mechanism for strong southward IMF, Ann. Geophys., 24, 381-392, 2006, http://www.ann-geophys.net/24/381/2006/.

Rijnbeek, R. P., Cowley, S. W. H., Southwood, D. J., and Russell, C. T.: A survey of dayside flux transfer events observed by ISEE 1 and 2 magnetometer, J. Geophys. Res., 89, 786-800, 1984.

Russell, C. T. and Elphic, R. C.: Initial ISEE magnetometer results: Magnetopause observations, Space Sci. Rev., 22, 681-715, 1978

Russell, C. T., Berchem, J., and Luhmann, J. G.: On the source regions of flux transfer events, Adv. Space Res., 5, 363-368, 1985

Sonnerup, B. U. Ö.: Magnetopause reconnection rate, J. Geophys. Res., 79, 1546-1549, 1974.

Sonnerup, B. U. Ö., Hau, L.-N., and Walthour, D. W.: On steady field-aligned double-adiabatic flow, J. Geophys. Res., 97, 1201512028, 1992.

Swisdak, M. and Drake, J. F.: Orientation of the reconnection X-line, Geophys. Res. Lett., 34, L11106, doi:10.1029/2007GL029815, 2007.

Trattner, K. J., Fuselier, S. A., and Petrinec, S. M.: Location of the reconnection line for northward interplanetary magnetic field, J Geophys. Res., 109, A03219, doi:10.1029/2003JA009975, 2004.

Wang, Y. L., Elphic, R. C., Lavraud, B., et al.: Initial results of high-latitude magnetopause and low-latitude flank flux transfer events from 3 years of Cluster observations, J. Geophys. Res., 110, A11221, doi:10.1029/2005JA011150, 2005. 